\title{
SISTEMA INTEGRADO DE GESTIÓN DE CALIDAD AMBIENTAL SUSTENTABLE PARA LA ZONA ECUATORIAN ANDINA
}

\author{
M.B.B.Avalos ${ }^{1 *}$; I.G.Rondón ${ }^{2}$; S.P.B.Avalos ${ }^{3}$ e Z.L.R.Cotilla ${ }^{2}$ \\ 1 Escuela Superior Politécnica de Chimboraz, EC060155, Riobamba, Ecuador. \\ 2 Universidad de La Habana, Havana, Cuba \\ 3 Universidad de Las Américas, Santiago, 72819, Región Metropolitana, Chile \\ * belen_b33@hotmail.com
}

Artigo submetido em 25/07/2016 e aceito em 25/08/2016

\section{RESUMEN}

El crear un sistema que integre la gestión de calidad y la gestión ambiental es de suma importancia en la actualidad, basado en epistemología, normas y sistemas existentes con características similares que permiten su integración pero lo que llama la atención de esta metodología es que no es únicamente para empresas privadas, públicas de comercialización, de servicios o productos; sino más bien es para zonas naturales que son los espacios con mayor afectación; hasta la actualidad su desgaste va en crecimiento constante; esto a lo largo del tiempo ha afectado y sigue afectando la sustentabilidad, y por ende desmejorando la calidad de vida de la población.
La finalidad de este artículo es sociabilizar el sistema que integra en uno solo los sistemas de gestión según las necesidades de las zonas naturales, con la única finalidad de mejorar su gestión, poder mantener y mejorar la sustentabilidad de la zona seleccionada donde se aplique el sistema integrado, a fin de mejorar la calidad de vida de la población de dicha zona natural. Los indicadores que integra este sistema, permitirán tener un frecuente análisis comparativo, mismo que coadyuvará a la toma de acciones preventivas y/o correctivas, así como también adoptar políticas de mejora continua.

PALAVRAS-CHAVE: Gestión de calidad, gestión ambiental, integrado, sistemas, zonas naturales.

\section{INTEGRATED MANAGEMENT SYSTEM FOR ENVIRONMENTAL QUALITY OF THE ANDINA ECUATORIAN AREA}

\begin{abstract}
Creating a system that integrates quality management and environmental management is paramount today, based on epistemology, standards and systems with similar features that allow integration but what draws the attention of this methodology is that it is not only for private companies, public marketing, services or products; but rather it is to natural areas that are most affected areas; until now wear is constantly growing; This over time has affected and continues to affect sustainability, and therefore spoiled the quality of life of the population. The purpose of this article is to socialize
\end{abstract}

the system integrates into one management systems according to the needs of natural areas, with the sole purpose of improving their management, to maintain and improve the sustainability of the selected area where the system is applied integrated to improve the quality of life of the population of this natural area. The indicators that integrates this system will allow to have a common comparative analysis, same as will contribute to taking preventive actions and / or corrective actions, as well as adopt policies of continuous improvement.

KEYWORDS: Quality management, environmental management, integrated systems, natural areas. 


\section{INTRODUCCIÓN}

La teoría general de sistemas abreviada por sus siglas como TGS, surge como un aporte, gracias a la necessidade de conceptos y leyes válidas para la descripción e interpretación de toda clase de sistemas. Bertalanffy Ludwing y Katz Kuhn que hasta la actualidad y principalmente el primer autor es considerado como el padre de la teroría de los sistemas, fueron ellos em el año de 1966, los primeros en aplicar una teoría de sistemas, luego se su aplicación, concluyen que los sistemas son mecanismos de entrada, producción y salida. Las entradas se refieren al ambiente transformado, pudiendo ser en forma de energía, información, dinero, personas, materias primas, entre otras; cada uno de estos debe funcionar adecuadamente para que el sistema sea efectivo; todos los sistemas tienen propósitos y metas, convirtiéndose éstas en las razones de su existencia.

En torno a esto, la planificación de los sistemas abiertos se refiere a examinar el ambiente con el objeto de determinar otras expectativas que tienen las organizaciones, espacios o las zonas naturales entre ellas mismas, siempre y cuando tengan características similares.

La TGS propuesta por el que es conocido como padre de esta teoria, busca reglas de valor general, aplicables a cualquier sistema y en cualquier nivel de la realidad cultural, social; surge por la necesidad de abordar científicamente la comprensión de los sistemas concretos que forman la realidad sobretodo la natural, generalmente complejas y únicas, pese a su similitud. Según este mismo autor, existen cuatro fundamentos de esta teoria que se acoplan a la realidad de las zonas naturales con características andinas. el primero innvestigar el isomorfismo de conceptos, leyes y modelos en varios campos y promover transferencias útiles de un campo a otro, el segundo favorecer el desarrollo de modelos teóricos adecuados en aquellos campos donde faltaran (zonas naturales andinas), el tercero reducir en lo posible la duplicación de esfuerzo teórico en campos distintos (integración de la calidad y el ambiente), y el cuarto y último promover la unidad de la ciencia, mejorando la comunicación entre los especialistas.

Con el pasar del tiempo varios autores según el análisis de Kuhn (1996), apoyan el enfoque de sistemas donde dice que es la base para la aplicación de estrategias de productividad, dado que un sistema es una estructura organizada y unitaria, compuesta de dos o más elementos o partes interdependientes, componentes o subsistemas delineados por límites identificables que lo separan de su supra sistema ambiental.

Al tomar estos conceptos y concordando com el critério de Kuhn los autores de esta investigación concluyen que la necesidad de un sistema es imperante en la actual economía a pesar que varios años atrás ya se conocía teóricamente por algunos precursores, muchos de los economistas clásicos y neoclásicos rechazaron estas afirmaciones hasta que ahora en el siglo actual palpamos y vivimos en este mundo con problemas socioeconómicos debido a la degradación ambiental; su calidad ha disminuido notablemente afectando de manera directa la calidad de vida del mundo entero.

Se propone un sistema integrado de gestión de calidad ambiental basado en la sustentabilidad con la finalidad de mejorar la calidad de vida de la población y cuidar el lugar en donde habitamos, consumimos y producimos de tal manera que las generaciones que vendrán puedan seguir disfrutando de las mismas riquezas naturales de las que actualmente podemos disfrutar física y visualmente.

\section{MATERIALES Y MÉTODOS}

A partir del análisis en este punto, la investigación necesita de una adecuada gestión para poder hablar de sustentabilidad que más adelante la definiré. Pero es imperioso iniciar indicando 
que es gestión. La palabra gestión proviene del vocablo original en inglés management (administración); apoyada en la teoría de los autores como Dourojjeanni, Hubel, Anthony R.N, se pretende matizar las dos acepciones (administración y gestión) dando a la gestión un carácter mucho más amplio y general, puesto que durante el siglo XX desfilaron, ante las empresas, una gran variedad de enfoques de gestión, así por ejemplo, los años 50 se caracterizaron por un enfoque hacia la producción, en los años 60 el enfoque se centró en la mercadotecnia, los años 70 trajeron un enfoque hacia la gestión estratégica, los años 80 llegaron con una gestión basada en la búsqueda de una alta calidad de los productos y/o servicios y con tendencia a la reingeniería de procesos.

Basada en esta tendencia y evolución de la definición de gestión es indispensable conocerla para hablar de un sistema integrado, que por sí solo habla ya de una calidad; para evitar contradicciones y al investigar pude descubrir que Pérez $\mathrm{C}$, hace el análisis de 62 conceptos a través de un dendograma obteniendo como definición de gestión como "un proceso dinámico, interactivo, eficiente y eficaz; consistente en planear, organizar, liderar y controlar las acciones en la entidad, desarrollado por un órgano de dirección que cuenta con grupos de personas, recursos y autoridad para el establecimiento, logro y mejora de los propósitos de constitución de la organización, sobre la base del conocimiento de las leyes y principios, de la sociedad, la naturaleza humana y la técnica, así como de información en general" (Pérez Campdesuñer, 2006), corrobora la teoría de los autores y entonces defino a gestión como el conjunto de acciones orientadas al logro de los objetivos y metas a través de procedimientos, es decir, la gestión implica el desarrollo amplio de las funciones de planificar, hacer, verificar, y actuar de determinadas actividades.

Hasta aquí hemos definido los sistemas y la gestión, pero nos queda la interrogante ¿Qué relación existe entre estas dos definiciones y la calidad?, El término calidad ha sido objeto de debate desde la antigüedad hasta la actualidad, desde Cicerón, Aristóteles, Kant, Hegel, Engels, Lenin, Kursanov, Kusinen, entre otros han intentado un acercamiento al concepto. En la literatura especializada sobre calidad se pueden encontrar definiciones desarrolladas por diversos autores, entre ellos Deming (1989), Feigenbaum (1986), ISO 8402 (1986), Yamaguchi (1989), Crosby (1992), Jurán (1993), ISO 8402 (1996), ISO 9000 (2000), Michelena Fernández (2000) y Moreno Pino (2003) además de ASQC, Tuchman, Ruskin y Drucker, 2004. Tomando el concepto de Paneque Reyes (2001) donde manifiesta que "la Calidad es un concepto en el que los teóricos del tema no se han puesto de acuerdo, cada uno tiene su propia concepción, todas son correctas, pero enfocan en diferentes aspectos. Y es que el campo de aplicación de la calidad es tan amplio, que es imposible tener una sola frase que cubra todas las posibilidades". Al realizar un análisis comparativo de los diferentes conceptos emitidos en virtud de encontrar las tendencias en el desarrollo conceptual concluyo que calidad es el conjunto de atributos esenciales que propician la satisfacción de las necesidades.

\section{SISTEMAS DE GESTIÓN DE CALIDAD VS. SISTEMAS DE GESTIÓN AMBIENTE}

Diversos autores opinan que, dentro de la gestión general de cualquier organización, se debe establecer claramente la estructura de cada uno de los sistemas de gestión particulares y subsecuentemente del sistema de gestión. Esto incluye definir claramente la estructura organizativa, como sus procesos, y como llevarlos a cabo, procedimientos mediante los cuales se ejecuta las actividades y las tareas, así como establecer los recursos de los cuales se dispone. (Ackoff, 2007)

Se ha descrito en forma armónica los componentes de la estructura organizativa, también se hace hincapié en que las diversas partes del sistema de gestión de una organización deben integrarse en un sistema de gestión único, coherente y unificado que utilice elementos comunes. Esto facilita 
la planificación, la asignación de recursos, el establecimiento de objetivos complementarios y la evaluación de la eficacia.

Los sistemas de gestión, sea en forma individual o integrada, deben estructurarse y adaptarse al tipo y las características de cada organización (finalidad o actividad), tomando en consideración particularmente los elementos que sean apropiados para su estructuración, para lo cual se debe definir claramente los siguientes aspectos:

1. La estructura organizativa (incluyendo funciones, responsabilidades, líneas de autoridad y de comunicación),

2. Los resultados deseables que se pretende lograr,

3. Los procesos que se llevan a cabo para cumplir con la finalidad,

4. Los procedimientos mediante los cuales se ejecuta las actividades y las tareas

5. Los recursos con los cuales se dispone.

Los sistemas de gestión se aplican en el marco de todas las actividades que se ejecutan en una organización, zona o área y son válidos solo si cada uno de ellos interactúa con los demás armónicamente.

Ahora bien, al saber que las dos concepciones de gestión y calidad tienen puntos comunes en sus variables conceptuales y se los puede unificar entonces se plantea que la gestión de la calidad es: un proceso consistente en planear, hacer, verificar y actuar para el logro de un conjunto articulado de atributos, que determinan el grado en que los procesos que logran propiciar la satisfacción de la sociedad. Entonces concluyo que la gestión de calidad es el conjunto de controles adecuados en una organización, zona o área supeditados entre sí, con los cuales la organización podrá administrar de modo organizado la calidad de la misma. La misión siempre estará enfocada hacia la mejora continua de la calidad.

La estructura de los sistemas de gestión de calidad debe ser tal que sea factible realizar una coordinación, un control ordenado y permanente sobre la totalidad de las actividades que se realizan considerando sus principios. Al querer realizar una integración entre un sistema de gestión de calidad y un sistema de gestión ambiental, primero es imperante entender que es ambiente para lo cual busco su definición, que en la actualidad es considerado como un sistema global constituido por elementos naturales y artificiales, físicos, químicos o biológicos, socioculturales y sus interacciones, en permanente modificación por la naturaleza o la acción humana, que rige la existencia y desarrollo de la vida en sus diversas manifestaciones (Ley de Gestion Ambiental, 2004). Según el (Texto Unificado Legislación Secundaria Medio Ambiente, 2003) manifiesta que el ambiente comprende los alrededores en los cuales la organización opera, incluye el agua, aire, suelo, recursos naturales, flora, fauna, seres humanos, y su interrelación.

La gestión ambiental se hace necesaria en el siglo XXI, debido a que persisten las desigualdades sociales, mismas que permiten a unos pocos explotar en su beneficio los recursos ambientales y alejar tanto temporal como espacialmente las consecuencias dañinas de sus acciones no sostenibles; por otro lado, estas mismas desigualdades ocasionan que los grupos más desfavorecidos soporten de forma más inmediata y en sus entornos más próximos las consecuencias negativas de este modelo de desarrollo no sostenible y que dada su debilidad estructural les sea muy difícil iniciar cambios en el modelo económico que distribuyan equitativamente y, en última instancia, eliminen las externalidades ambientales.

La gestión ambiental debe permitir mantener un conjunto de políticas, normas, actividades operativas y administrativas de planeamiento, financiamiento y control estrechamente vinculadas, 
que deben ser ejecutadas por el estado y la sociedad para garantizar el desarrollo sustentable y una óptima calidad de vida. (Ley de Gestion Ambiental, 2004)

Ante la situación planteada el ambiente abarca factores físicos naturales, perceptuales y socioeconómicos, inherentes a la presencia del ser humano. Si se considera al hombre independiente del ambiente, la preocupación por el deterioro ambiental sería infundada, sería el cuidado del ambiente por el ambiente y no el ambiente por una calidad de vida del hombre; el ambiente implica directamente al hombre en el ámbito espacial, social y económico. El ambiente es el lugar en donde el hombre se desarrolla fisiológica, social y económicamente, interactuando con los recursos existentes.

En correspondencia con lo expuesto las normas ISO 14001:2004 establece que la definición para un Sistema de Gestión Ambiental "es la parte del sistema de una organización empleada para desarrollarse e implementar su política ambiental y gestionar sus aspectos ambientales, incluyendo la estructura de la organización, la planificación de actividades, las responsabilidades, las prácticas, los procedimientos, los procesos y los recursos"

La Unidad de Planeación, Minero, Energético de Colombia, Bogota D.C. emite el concepto de Sistema de Gestión Ambiental: "Sistema de Gestión Ambiental, es un conjunto de acciones encaminadas a lograr la máxima racionalidad en el proceso de decisión, relativo a la conservación, defensa, protección y mejora del medio ambiente, basándose en una coordinada información multidisciplinaria y en la participación ciudadana (Esteban Bolea 1994)

Con lo expuesto en cuanto a su definición para un Sistema de Gestión Ambiental, y de acuerdo a diversas opiniones a nivel mundial, se establece que se trata de la planificación y realización de actividades organizadas con el objetivo de proteger el medio ambiente, lo cual se traduce en el mejoramiento de la calidad de vida humana, considerando temas sociales, económicos, culturales entre otros, sin olvidar la premisa del Informe Brundtland" o "Nuestro Futuro Común", que el mejoramiento de la calidad de vida permite satisfacer las necesidades de las generaciones presentes sin comprometer la capacidad de las futuras generaciones para satisfacer las propias.

\section{RESULTADOS Y DISCUSIÓN}

\subsection{El sistemas de gestión de calidad y de gestión ambiental en las zonas naturales andinas ecuatorianas.}

Aunque el Ecuador posee una gran biodiversidad, actualmente presenta un panorama ambiental desconcertante, con preocupantes indicadores como una alta tasa de deforestación y erosión de los suelos, pérdida creciente de la biodiversidad y de los recursos genéticos, desordenada e irracional utilización de los recursos naturales, creciente contaminación del agua, aire y suelo, deficiente manejo de desechos, desertificación, sequías e inundaciones, deterioro de las cuencas hidrográficas, deterioro de las condiciones ambientales humanas, además de inequidad en la distribución de la riqueza (Fundación Natura, 20012).

Alrededor del $25 \%$ de todas las enfermedades prevenibles son causadas principalmente por la degradación del ambiente, la mala gestión de los recursos naturales, la excesiva producción de desechos orgánicos y los patrones de producción en los países (OPS, 2010). En la actualidad no se trata solamente de conservar y proteger la naturaleza para el desarrollo, sino de construir nuevos estilos de vida que permitan la manifestación de lo diverso, en lo cultural y en lo natural, y la realización de potencialidades individuales y colectivas.

A pesar de los esfuerzos realizados no se ha logrado un verdadero aprovechamiento sustentable de los recursos hasta hoy en día; la vegetación nativa ha disminuido considerablemente 
y por el contrario, la población sigue creciendo y la contaminación del agua aire suelo se ha incrementado, afectando la calidad de vida de la población como se muestra en la gráfica 1.

Gráfica 1 - Crecimiento poblacional del Ecuador

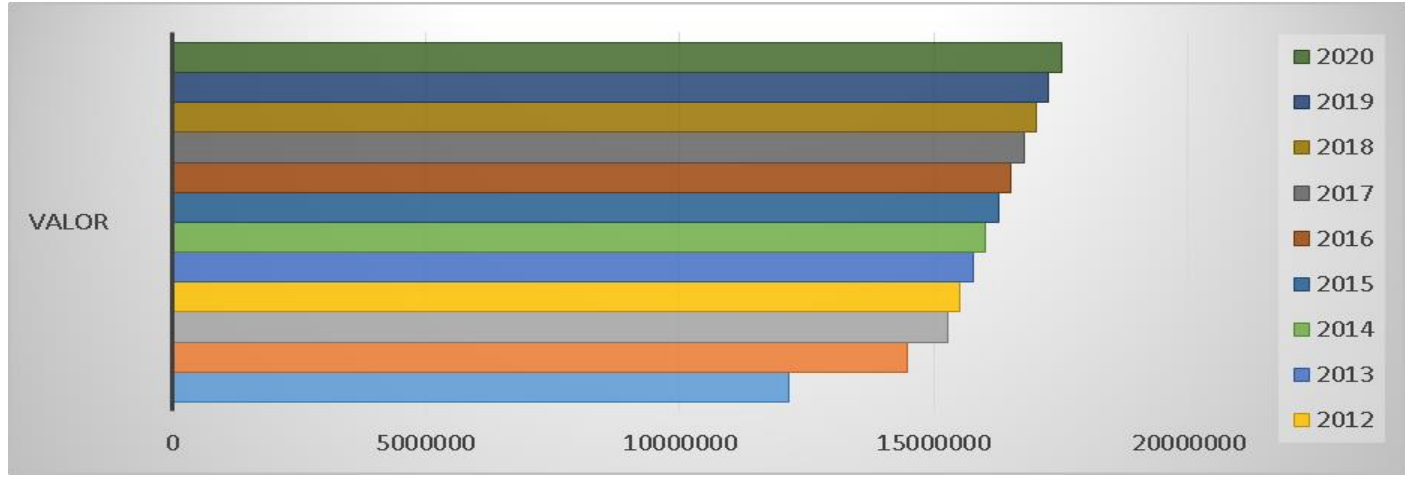

Fuente: Instituto Nacional Ecuatoriano de Censos

Es así que el término desarrollo sostenible se comenzó a utilizar en la primera estrategia para la conservación en 1980, dada la evidencia que el "desarrollo" había generado una gran cantidad de impactos ambientales negativos y que se necesitaba otro modelo de desarrollo que superara esos efectos indeseables. De esta manera las Naciones Unidas en el Informe Brundtland en 1987, definen al desarrollo sostenible como: "el desarrollo que satisfaga las necesidades del presente sin poner en peligro la capacidad de las generaciones futuras para atender sus propias necesidades".

El desarrollo sostenible, está dirigido al cambio de las modalidades de producción consumo y distribución de los recursos naturales e implica entre otros un rediseño de las formas tradicionales de producción altamente contaminantes y destructoras del ambiente; entonces puedo afirmar que el desarrollo sostenible actualmente aspira crear relaciones más sólidas entre los aspectos sociales económicos y ambientales. Por tanto, existe relación directa entre el riesgo de degradación del ambiente y el nivel de afectación a las poblaciones en general y de manera especial a las más pobres.

En este punto podemos hablar de integración, la cual es una forma eficaz de ahorrar costos, mejorar la comunicación y obtener una mayor igualdad en la estrategia planteada; empieza a notarse la necesidad de integrar estos dos sistemas como se observa en la figura 1. El concepto del sistema de gestión integrado surge como una forma en que puede asegurarse de que se cumpla de manera eficaz y eficiente con requisitos de varios sistemas de gestión. Así la integración de los sistemas puede tener ventajas como:

- Satisfacer las necesidades y expectativas de todas las partes interesadas al establecer la obligatoriedad de cumplir al mismo tiempo con los requisitos legales y regulatorios, los ambientales, de calidad.

- Mejorar la eficacia y la eficiencia de los procesos, aumentando la consistencia, la trazabilidad, evitando las redundancias y las incoherencias.

- Se integran los procesos de planificación de objetivos y revisión de un solo sistema ya que la definición de los objetivos del sistema de Gestión Integrado abarca objetivos ambientales y de calidad.

- Solucionar conflictos de responsabilidades, optimizar y simplificar el proceso de toma de decisiones basadas en datos integrales puesto 
- Alcanzar una mayor coherencia, facilidad de manejo y reducción de los costos de mantenimiento de la documentación al integrarse en un solo documento (procedimiento, instrucción, registro, etc.) aspectos relacionados con las dos funciones.

- Incrementar el rendimiento, las competencias y el entrenamiento de la población de la zona, ya que se determinan y suplen al unísono las necesidades de formación de los individuos en varias áreas.

- Ahorrar recursos en el desarrollo e implementación del Sistema de Gestión Integrado y una menor inversión que la necesaria para los procesos de certificación de estos sistemas independientemente.

- Se pueden auditar al mismo tiempo los dos sistemas, con lo cual se reducirán los costos que supone la preparación y realización de controles (auditorías).

- Aprovechar la experiencia en sistemas existentes para la implantación de otros.

Con esta integración, figura 1 , de los aspectos sociales económicos y ambientales pueden dar como resultado un desarrollo sustentable. El término sustentabilidad sufrió diferentes transformaciones a lo largo del tiempo hasta llegar al concepto moderno basado en el desarrollo de los sistemas socio ecológicos para lograr una nueva configuración en las tres dimensiones centrales del desarrollo sustentable: la económica, la social y la ambiental, pero empecemos por el principio ¿qué se entiende por un proceso sostenible?

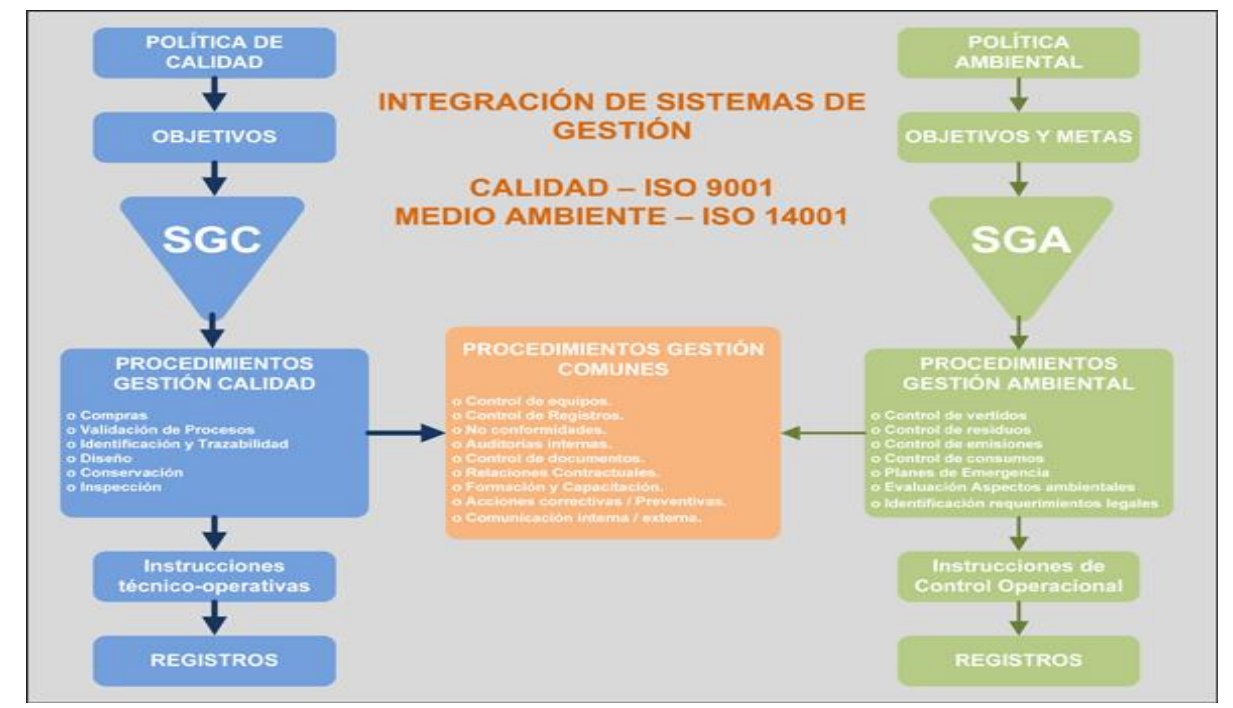

Figura 1- Integración ISO 9000 - ISO 14000

Fuente- Adaptado de Boletín Calidad\&Gestión No. 62

Los autores al indagar varios autores puede acordar que un sistema o proceso como sostenible de la siguiente manera, "Un proceso es sostenible cuando ha desarrollado la capacidad para producir indefinidamente a un ritmo en el cual no agota los recursos que utiliza y que necesita para funcionar y no produce más contaminantes de los que puede absorber su entorno." Luego de esta pequeña intervención es necesario hablar acerca de la definición de sustentabilidad.

El concepto de la sustentabilidad parte de un fundamento básico, pero comprometedor, "El desarrollo sustentable hace referencia a la capacidad que haya desarrollado el sistema humano para satisfacer las necesidades de las generaciones actuales sin comprometer los recursos y oportunidades para el crecimiento y desarrollo de las generaciones futuras." Esta definición se 
expresó por primera vez, haciendo referencia al desarrollo sustentable, en el informe Brundtland, "Our Common Future", publicado en 1987. Luego de ese informe quedó bautizada la definición y es la que más aceptación tiene en toda la comunidad cuando se habla de desarrollo sustentable. En la actualidad el World Commission on Environment and Development de las Naciones Unidas adoptó esta definición para desarrollo sustentable.

Suecia, uno de los líderes actuales en sustentabilidad tiene una definición un poco más holística y define una sociedad sustentable como: "Una sociedad en la cual el desarrollo económico, el bienestar social y la integración están unidos con un medioambiente de calidad. Esta sociedad tiene la capacidad de satisfacer sus necesidades actuales sin perjudicar la habilidad de que las generaciones futuras puedan satisfacer las suyas".

A más detalle esta definición desde el punto de vista de la prosperidad económica, queda expresado de la siguiente manera, "Sustentabilidad es la habilidad de lograr una prosperidad económica sostenida en el tiempo protegiendo al mismo tiempo los sistemas naturales del planeta y proveyendo una alta calidad de vida para las personas." Esta definición representa el concepto moderno de sustentabilidad; sin embargo, desde diferentes ámbitos, existe una marcada tendencia a considerar que esta definición tiene contradicciones en sí misma. Plantean que buscar el "equilibrio" entre ambiente, economía y sociedad perjudicará el progreso económico.

Por ello, un aspecto significativo relacionado con el término sustentabilidad es su posición frente a lo que entendemos como progreso económico. La confusión radica en que muchas veces se confunde a la sustentabilidad con volver a estados anteriores primitivos. Para estar en armonía con la naturaleza muchas personas piensan que la sustentabilidad y el progreso no son cosas que vayan de la mano. No obstante es interesante entender que esencialmente se busca a partir de la sustentabilidad es avanzar hacia una relación diferente entre la economía, el ambiente y la sociedad como se muestra en la figura 2.; no busca frenar el progreso ni volver a estados primitivos, todo lo contrario busca precisamente promover un progreso pero desde un enfoque diferente y más amplio, y ahí es donde reside el verdadero desafío.

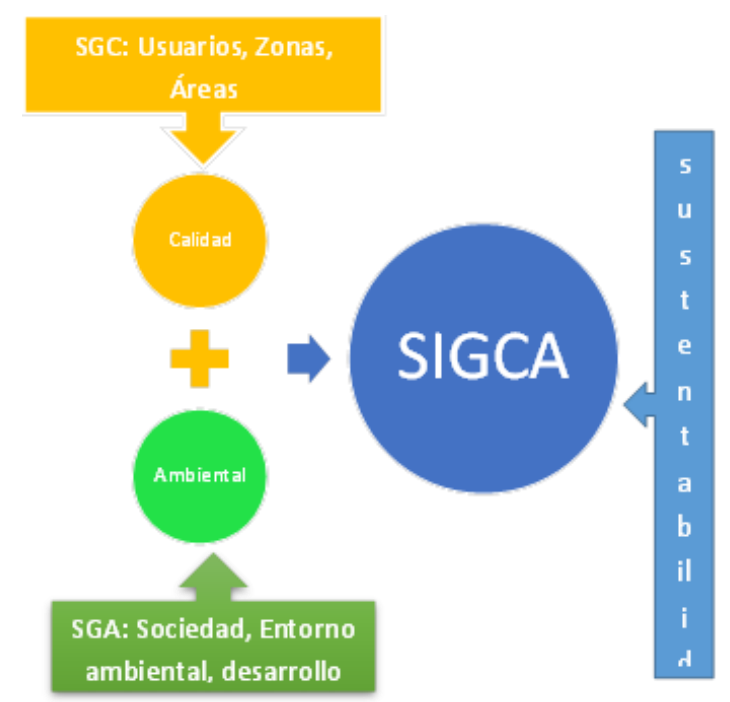

Figura 2- Integración del Sistema de Gestión de Calidad y el Sistema de Gestión Ambiental, basado en la sustentabilidad

Esto implica no sólo un cambio en ciertas prácticas y procedimientos para un nuevo paradigma acerca de la relación entre nuestra experiencia cotidiana, nuestro entorno y nosotros mismos, así la sustentabilidad es un proceso integral que exige a los distintos actores de la sociedad 
compromisos y responsabilidades en la aplicación del modelo económico, político, ambiental y social, así como en los patrones de consumo que determinan la calidad de vida.

\subsection{Creación de un Sistema Integrado de Gestión de Calidad Ambiental Sustentable (SIGCAS).}

Como se observa en la figura 3 el sistema que se propone, tiene cuatro enfoques:

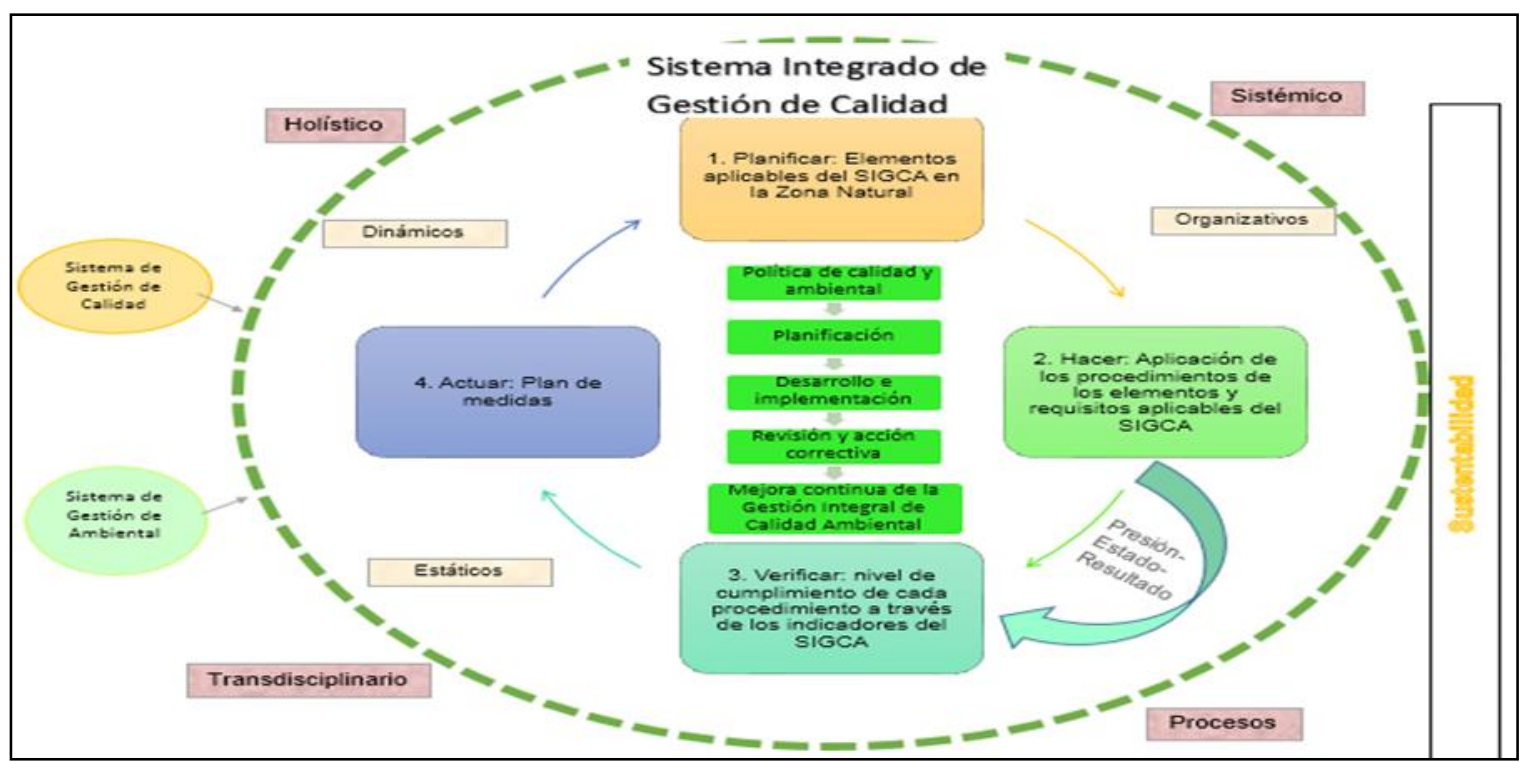

Figura 3- Sistema Integrado de Gestión de Calidad Ambiental Sustentable.

El enfoque sistémico porque es posible reconocer estructura de la organización, zona o área, las responsabilidades, los procedimientos, los procesos y los recursos que se establecen para llevar a cabo la gestión integrada, es decir de la totalidad, lo cual establece que, debido a sus relaciones internas, cualquier acción en los requisitos del Sistema Integrado de Gestión de Calidad Ambiental Sustentable (De aquí en adelante lo llamaremos SIGCAS) o la errónea aplicación de un procedimiento traerá consigo la afectación de todas las partes.

De igual manera, su enfoque holístico se confirma a través los procesos de gestión dependerán, fundamentalmente, para implementar el SIGCAS se debe considerar los requisitos, sin embargo, podrían resumirse en 5 grupos básicos, figura 4, tal como se muestra en la figura No. 1, mientras que su enfoque transdisciplinario debido a la Integración que realiza de calidad, el ambiente y el factor socio económico dando respuesta a la sustentabilidad.

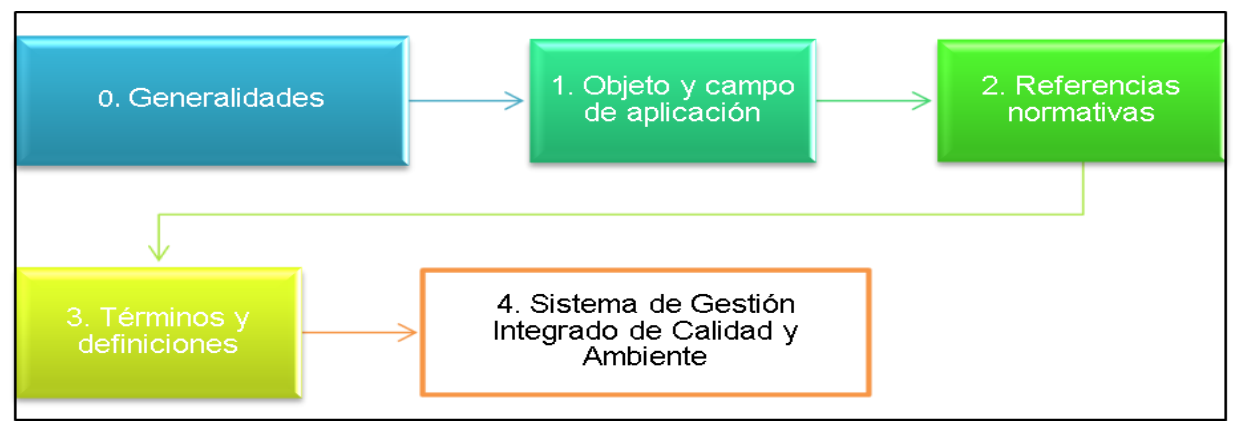

Figura 4- Grupos del SIGCAS.

Fuente: Elaboración Propia a Partir de Sistema de Gestión de Calidad y Sistema de Gestión Ambiental 
El sistema se caracteriza por realizarse con una lógica de procesos que en un orden predeterminado están orientados al logro de un objetivo específico. La base de este sistema implica el desarrollo de un proceso dinámico que sigue el ciclo planteado por Deming y vigente hasta la actualidad aplicada también por la norma internacional ISO 9001:2008. El SIGCA propone requisitos los cuales se podrían agrupar en 5 principales grupos como se describen en la figura 4 .

Sin embargo, en el requisito 4, Sistema de Gestión Integrado de Calidad Ambiental Sustentable, se determinan los subrequisitos para poder ejecutarlo, mismo que se desarrolla en 5 grupos principales, graficados en el centro de la figura No.1 y en la figura 5

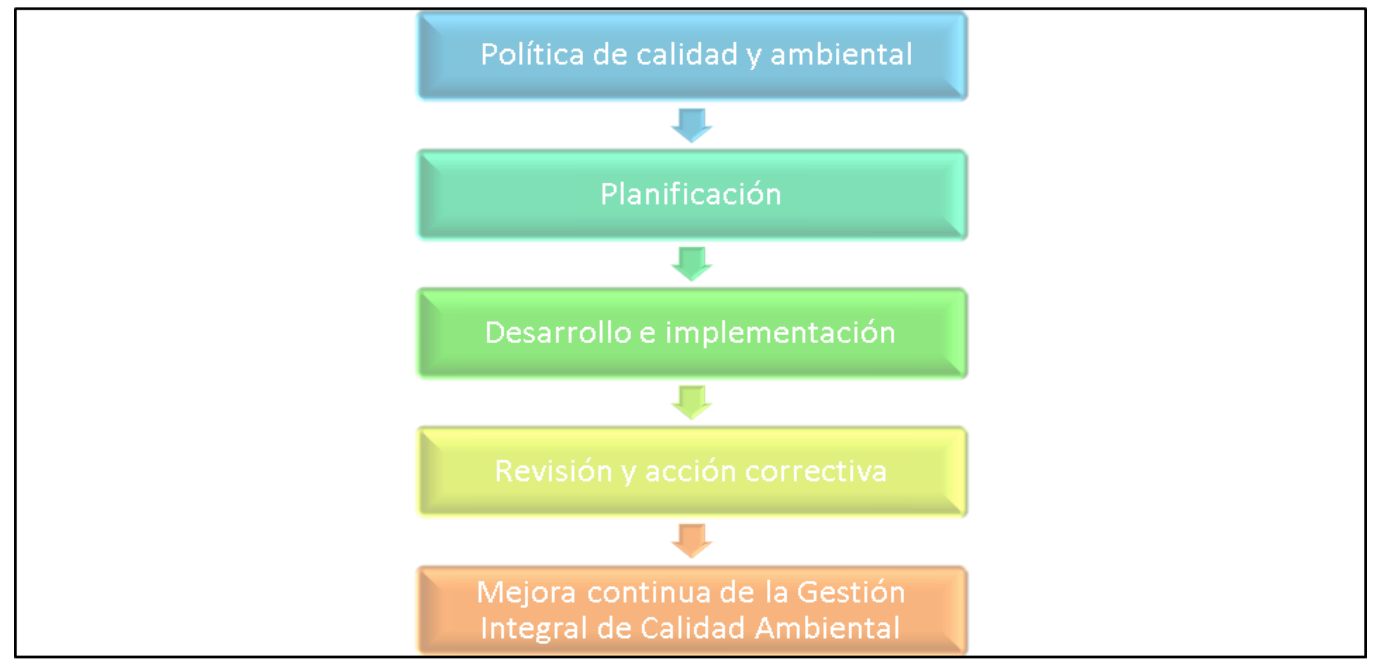

Figura 5- Requisitos del SIGCAS

Fuente: Elaboración Propia a Partir de los requisitos del SIGCAS

El SIGCAS, de acuerdo al esquema presentado, está conformado por 5 grupos de requisitos necesarios; su intención es enfocar los esfuerzos hacia el mejoramiento continuo de las acciones y resultados que adopte el sistema, mediante un proceso cíclico de planificación, ejecución, verificación y mejora continua. La consecución de ventajas de un SIGCAS para organizaciones, zonas o áreas, requiere del cumplimiento de ciertos aspectos importantes:

- Cumplir con los requisitos legales aplicables a las actividades de la zona natural

- Establecer una política sustentable.

- Identificar los aspectos de gestión, económicos, ambientales y sociales relacionados con las actividades normales de la zona natural

- Identificar los requisitos legales pertinentes nacionales y locales.

- Identificar las propiedades y establecer los objetivos sustentables pertinentes.

- Facilitar la planificación, el control, la supervisión, las acciones correctivas, las auditorias y las revisiones que garanticen el cumplimiento de la política sustentable

- La implementación de indicadores que permitan medir el índice de sustentabilidad

- Tener la capacidad de adaptarse a posibles modificaciones.

Cada uno de los requisitos del SIGCAS propuesto tiene procedimientos que explican su funcionalidad y ayudan al cumplimiento de ellos al momento de ser aplicados. Los aspectos organizativos son los referidos a la descripción de la organización, zona o área y también a la 
preparación del sistema. Definen los procesos que han de llevarse a cabo para que cumpla sus fines, los objetivos que debe alcanzar y la forma como está estructurada la organización, zona o área, los cuadros organizativos socialmente, así como las condiciones de competencia, nivel de formación y las relaciones de comunicación internas.

Los aspectos dinámicos contemplan la preparación y ejecución de los procesos y son característicos de la gestión de calidad, ya que definen las actividades de la población, tanto en las actividades diarias (realización de los trabajos) como en el control de los resultados. Los aspectos estáticos son característicos de la gestión ambiental describen fundamentalmente la situación en que deben encontrarse las zonas naturales a fin de que sean sustentables para las actuales y futura poblaciones, así como también para el entorno circundante y las protecciones que han de ser utilizadas para mantener o aumentar la sustentabilidad.

Cada uno de los principios del SIGCAS constituye un elemento que permite lograr competitividad y sustentabilidad de la zona natural donde se aplique el sistema. Es importante determinar todos los procedimientos que se realizan, puesto que estos son la estructura ósea del sistema. Si vemos a los procedimientos como la estructura que mantiene erguido al SIGCAS en sus cuatro principales epígrafes. Es por eso que en el SIGCAS se detallan los requisitos a cumplir con su respectivo objetivo, que ayuda a identificar la razón del procedimiento a realizar y enfocar adecuadamente las actividades detalladas.

El direccionamiento del indicador para su aplicación, el tipo de indicador basado en la metodología PER (Presión, Estado, Resultado), explicado mediante la figura No. 4. Además para medir el nivel de gestión el SIGCA propuesto plantea un conjunto de indicadores para medir su cumplimiento basado en la metodología PER; metodología que se muestra en la figura 6.

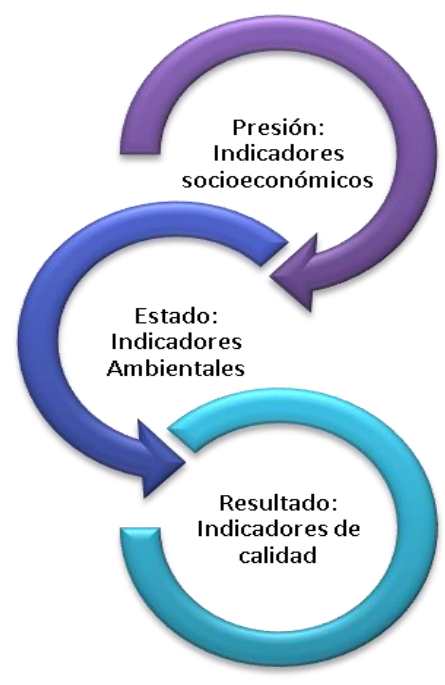

Figura 6- Modelo conceptual de la metodología PER

Fuente: Elaboración propia a partir del modelo CIAT

Para poder medir el cumplimiento de cada requisito tiene un objetivo que cumplir, también se detalla el procedimiento a realizar; una vez ejecutado el procedimiento se debe medir a través del indicador propuesto y sugerido con la finalidad de determinar si ese procedimiento cumplió su objetivo y el nivel de cumplimiento, según la unidad de medida que corresponda.

Para pasar a la última fase del sistema propuesto es decir al momento de Actuar el SIGCAS propone crear un plan de manejo ambiental, el cual deberá cumplir como mínimo con los pasos descritos en la figura 7. 


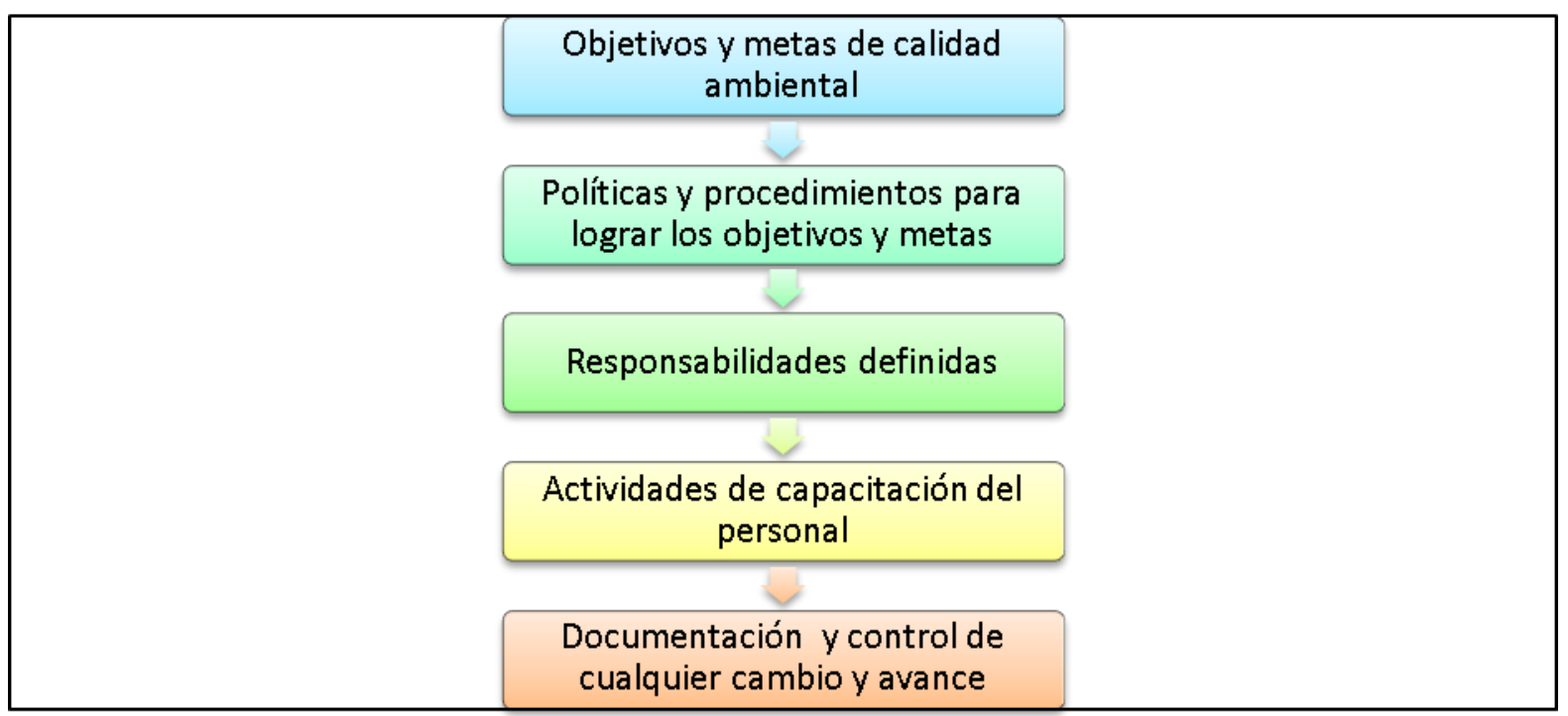

Figura 7- Proceso para la creación del plan de mejora continua

Fuente: Elaboración propia a partir de la ISO 14001:2004

\section{CONCLUSIÓN}

Al ser el SIGCAS replicable em otras organizaciones, zonas o áreas naturales los autores sugieren respetar las leyes ambientales que rigen para organización, zona o área natural, que sean objeto de aplicación del sistema propuesto.

Las causas generadoras de los problemas ambientales son siempre sociales; y las consecuencias de los mismos también recaerán sobre la sociedad; como premisa básica: el análisis socioeconómico es una poderosa herramienta para enfrentar los desafíos del desarrollo, este desarrollo principalmente económico, pero también cultural, social y humano.

El SIGCAS una vez aplicado en la zona natural mejorá la población en términos de calidad de vida del hombre, y ahorro vs gasto económico asociado a la actividad ecológica.

\section{REFERENCIAS}

AGUILERA GARCÍA, L.O. y ORTÍZ CHÁVEZ, Y. Los sistemas integrados de calidad y ambiente: gestión ambiental, ciencia, tecnología y equidad social en la educación universitaria. Innovación Educativa, v.12, n.59, p.103-120, 2012.

ALONSO PAULÍ, E. y CARRETERO, J.M. Sistemas Integrados de Gestión de calidad y medioambiente: evidencia empírica en la industria española". Cuadernos Económicos de ICE, v.86, p.1-11.

BERKES, F. et al. Navigating Social-Ecological Systems: BuildingResilience for Complexity and Change", Cambridge University Press, Inglaterra, 2001.

CALVENTE, Arturo. El concepto moderno de sustentabilidad, Universidad Abierta Interamericana, Buenos Aires, Argentina, 2010.

CEPAL. Políticas públicas para el desarrollo sustentable: la gestión integrada de cuencas, disponible en: http://www.cepal.org/publicaciones/xml/9/19759/lcr1399s.pdf, consulta: 27/4/2015.

COELLO, Y. Diseño del Sistema de Gestión Integrado Calidad-Medio Ambiente-Seguridad y Salud en el Trabajo, en el proceso Ejecución de Inversiones de la Empresa Eléctrica Holguín. Tesis para optar por el título de Ingeniero Industrial. 108 p., 2006.

FABREGAT, M., et al. Estrategia para la Formación de los Directivos en Gestión Ambiental. Contribución a la Educación y la protección ambiental. V.2, La Habana, Cuba, 2001. 
FERNÁNDEZ, A., et al. Política Económica, 4ta Edición, Editorial McGraw Hill, Madrid, España, 2005.
FLACSO
Andes,
Análisis
de
Coyuntura.
Disponible:

http://www.flacsoandes.org/web/imagesFTP/1333638283.Analisis_de_Coyuntura_Capitulo_1_Crecimiento_economico .pdf, Consulta: 16/52015, 2012.

GAINZA, E. integración de sistemas de gestión de calidad-medio ambiente y seguridad (INSIGE), disponible en: http://www.gestec.disaic.cu/tematicas.html, consulta 2008.

GÓMEZ, F. Situación actual y tendencia de los sistemas de gestión y la NTC -GP 1000/2004. Trabajo Presentado en la 12va semana de la Salud ocupacional. Colombia. 12 p., 2006.

Instituto Nacional de Estadísticas y Censos. Encuesta de Población y crecimiento poblacional). INEC, 2011.

IPARRAGUIRRE, A. La gestión compartida universidad-empresa em la formación del capital humano. Su relación com la competitividade y el desarrollo sostenible. Cuba. (2009).

ISO. Documento ISO/TC 176 Orientación acerca del enfoque basado en procesos para los sistemas de gestión de la calidad. $2001.10 \mathrm{p}$

ISO. Norma ISO 9001:2008. Sistema de Gestión de Calidad. Requisitos. 40 p., 2008.

ISO. Norma ISO 14001: 2004. Sistema de Gestión Ambiental. Requisitos con orientación para su uso. 26 p. Mayo, E. (1945), The social problems of an Industrial Civilization, Harvard University, Boston, EE.UU.

LLoyd's Register. Implementación del Sistema de Gestión de Calidad de acuerdo a los requisitos de ISO 9001:2000.Guía Práctica. 34 p.

MINISTERIO DEL AMBIENTE. Cuentas Nacionales de Huella Ecológica del Ecuador - Año 2012, 2015, Quito, Ecuador.

PEÑA. D. La integración de sistemas de gestión de la calidad, el medio ambiente y la seguridad y salud en el trabajo: Parte 4, 2010, disponible en: ww.gestiopolis.com, consulta: 17/3/2015.

Programa ONU - REDD. Documento del Programa Nacional - Ecuador. Sexta reunión de la Junta Normativa del Programa ONU - REDD, 2011, Da Lat, Vietnam.

Purdue University. Global Trade Analysis Project (GTAP). Disponible en: https://www.gtap.agecon.purdue.edu/, consulta: 9/8/2015.

RIVERO, Y. Diagnóstico de un Sistema de Gestión Integrado según los sistemas de gestión de la calidad, medioambiente y seguridad y salud ocupacional del trabajo en los procesos, de acuerdo a los requisitos de las normas en la UEB Proyecto y Construcción de la EEH. Tesis para optar por el título de Ingeniero Industrial. Universidad de Holguín, Cuba, 75 p., 2006.

RODRIGUEZ, M. y RICART, J. Coordinación de los sistemas de gestión de calidad, medio ambiente, salud laboral, Universidad de Navara, España, 2010.

Situación Ambiental Ecuador, anónimo, 2010.

TOR, D. Sistema Integrado de Gestión Ambiental - Seguridad y Salud Ocupacional, Libro digital, disponible en: http://www.bvsde.paho.org/bvsast/fulltext/gestion.pdf, consulta: 4/12/2015, 2003.

UN World Commission on Environment and Development (WCED). Our Common Future, Oxford: Oxford University Press, disponible en: http://www.un-documents.net/our-common-future.pdf, consulta: 12/9/2015, 1987.

VELÁZQUEZ, R. et al. "Los sistemas integrados de gestión. Un acercamiento teórico metodológico". Revista Ingeniare, v.5, n.3, p.18, 2008. 